\title{
Remarks on Carleman estimates and exact controllability of the Lamé system
}

\author{
Oleg Yu. Imanuvilov Masahiro Yamamoto
}

\begin{abstract}
In this paper we established the Carleman estimate for the two dimensional Lamé system with the zero Dirichlet boundary conditions. Using this estimate we proved the exact controllability result for the Lamé system with with a control locally distributed over a subdomain which satisfies to a certain type of nontrapping conditions.
\end{abstract}

\section{Introduction}

This paper is concerned with a Carleman estimates for the 2-D non stationary Lamé system with the Dirichlet boundary conditions. Starting from the pioneering works of Carleman the theory of Carleman's inequalities has been rapidly developed over the last forty years and now for a single partial differential equations many general results are available (see [Hö, ?, E2], [?].) On the other hand, for the systems of partial differential equations the situation is much less understood. To our best knowledge the most general result in case of systems of P.D.E. is the Calderon's uniqueness theorem (see e.g. [E1, Zui]). The technique, developed by Calderon reduces the system of partial differential equations to the system of pseudodifferential operators of the first order: $\frac{d v}{d x_{0}}=M\left(x, D_{x^{\prime}}\right) v+f$ where $M\left(x, D_{x^{\prime}}\right)$ is the matrix pseudodifferential operator. After that by some change of variables $v=Q\left(x, D_{x^{\prime}}\right) \tilde{v}$ this matrix P.D.O. $M$ will be reduced to $Q^{-1} M Q$ which consists of blocks of a small size located on the main diagonal, such that in each block the principal symbols of all operators located below the main diagonal are zero. In order, to construct the matrix $Q$ the eigenvalues and eigenvectors of the matrix $M\left(x, \xi^{\prime}\right)$ should be the smooth function of the variables $x$ and $\xi$ and eigenvalues should not change the multiplicity. This condition proved to be restrictive, especially in case when we are looking for a Carleman estimate near boundary, and therefore choice for a variable

The authors are deeply indebted to Prof. K. Yamamoto for the careful explanation of the results of the paper [Y2]. The first author supported partially by NSF Grant DMS-0205148.

Keywords : Carleman estimate, Lamé system. 
$x_{0}$ is limited. (For example the non stationary Lamé system in general it does not satisfy to this condition.)

On the other hand, it is well known that thanks to the special structure of the isotropic Lamé system $\operatorname{div} u, \operatorname{rot} u$ satisfy to scalar wave equations (modulo lower order terms.) The system of partial differential equations for a functions $u$, div $u$, rot $u$ is coupled via first order terms. This allows to apply the Carleman estimates for a scalar hyperbolic equations in case when the function $u$ has a compact support. (see [EINT]). There are many results on uniqueness of the Cauchy problem for the stationary Lamé system (see [DR, W1, W2]). Therefore the goal of this paper is to obtain the Carleman estimate for $u$ which does not vanish near boundary but satisfies only to the zero Dirichlet boundary condition. The structure of the proof is in principle similar to the paper [Y1]. We work with two hyperbolic equations for the functions $z_{\lambda+2 \mu}=e^{s \phi} \operatorname{div} u$ and $z_{\mu}=e^{s \phi} \operatorname{rot} u: P_{\lambda+2 \mu}(x, D, s) z_{\lambda+2 \mu}=(\operatorname{div} f) e^{s \phi}$ $; P_{\mu}(x, D, s) z_{\mu}=(\operatorname{rot} f) e^{s \phi}$. The main difficulty one should overcame is that there are no boundary conditions for these functions. This problem is fixed in following way. Microlocally, operators for $z_{\beta}$ could be decomposed ,expect for a small set in $T^{*}(Q)$, into the product of two pseudodifferential operators of the first order $P_{\beta}(x, D, s)=P_{-, \beta}\left(x, D_{x^{\prime}}, s\right) P_{+, \beta}\left(x, D_{x^{\prime}}, s\right)$ where $P_{ \pm, \beta}=D_{x_{2}}-\Gamma_{\beta}^{ \pm}\left(x, D_{x^{\prime}}, s\right)$, and $x_{2}$ is normal to the boundary $\partial \Omega$. Since the principal symbol of the operator $\Gamma_{\beta}^{-}\left(x, \xi^{\prime}, s\right)$ satisfies the inequality $-\operatorname{Im} \Gamma_{\beta}^{-}\left(x, \xi^{\prime}, s\right) \geq C^{2}|s|$ we have the a priori estimate for $\left.P_{+, \beta}\left(x, D_{x^{\prime}}, s\right) z_{\beta}\right|_{x_{2}=0}$ in $L^{2}$. These estimates combined with zero Dirichlet boundary condition, for stress tensor $u$ provide the $H^{1}$ boundary a priori estimates for $z_{\beta}$. The set on which the decomposition at least one of the operators $P_{\beta}(x, D, s)$ in the product of the first order operators is impossible is studied in sections 3,4 .

Among the applications of the Carleman estimate obtained in this paper first we mention the controllability results for the Lamé system. First controllability/observability results for the isotropic Lamé system with the constant coefficients were obtained by J.L. Lions in [Li] using the multiplies methods and HUM method. Later controllability and stabilizability properties for isotropic Lamé system and related models were studied by J.L. Lions and J. Lagnese in [LL] and [La]. Also we mention work [Y2], of K.Yamamoto where he studied the dissipation of the energy of Lamé system outside the convex obstacle. The results obtained in this paper could be easily converted in controllability results for the Lamé system using the HUM method. Recently, the technique developed in [Li] were applied by Alabau and Komornik [AK1, AK2] to prove the controllability/observability estimates were obtained for symmetric anisotropic Lamé system with the constant coefficients. In [B3], M. Bellassoued proved the approximate controllability for the isotropic Lamé system with the control distributed on an arbitrary small portion of the boundary.

Another possible application of the Carleman estimate is the inverse problem of the determination of the Lamé coefficients $\beta, \mu, \lambda$ using the finite number of measurements on a subdomain $Q_{\omega}$. (The corresponding problem when a finite number of measurements are available on the whole $[0, T] \times \partial \Omega$ was treated in [IIY].)

There are many papers concerning the uniqueness of the Cauchy problem for the Lamé system.( [DR, B3, W1]). The survey of resent results on the unique continuation for the stationary Lamé system given in [W2]. 


\section{Main Result}

Let us consider the 2-D Lamé system

$$
\begin{aligned}
& P u=\rho\left(x^{\prime}\right) \frac{\partial^{2} u}{\partial x_{0}^{2}}-\mu\left(x^{\prime}\right) \Delta u-\left(\mu\left(x^{\prime}\right)+\lambda\left(x^{\prime}\right)\right) \nabla \operatorname{div} u \\
& \quad-\operatorname{div} u \nabla \lambda\left(x^{\prime}\right)-\left(\nabla u+(\nabla u)^{T}\right) \nabla \mu\left(x^{\prime}\right)=f \quad \text { in } Q=[0, T] \times \Omega, \\
& \left.u\right|_{(0, T) \times \partial \Omega}=0, \quad u\left(T, x^{\prime}\right)=u_{x_{0}}\left(T, x^{\prime}\right)=u\left(0, x^{\prime}\right)=u_{x_{0}}\left(0, x^{\prime}\right)=0,
\end{aligned}
$$

where $u=\left(u_{1}, u_{2}\right), f=\left(f_{1}, f_{2}\right)$ are the vector functions, $\Omega$ is a bounded domain in $\mathbb{R}^{2}$ with $\partial \Omega \in C^{3}, x=\left(x_{0}, x^{\prime}\right), x^{\prime}=\left(x_{1}, x_{2}\right)$. Coefficients $\rho\left(x^{\prime}\right), \mu\left(x^{\prime}\right), \lambda\left(x^{\prime}\right) \in C^{2}(\bar{Q})$ are assumed to be the positive functions

$$
\rho\left(x^{\prime}\right)>0, \quad \mu\left(x^{\prime}\right)>0, \quad \lambda\left(x^{\prime}\right)>0 \quad \text { in } \bar{\Omega} .
$$

The goal of this paper is to establish a Carleman estimate for a system (1), (2). Let $\omega \subset \Omega$ be an arbitrary fixed subdomain. Denote by $\nu$ the outward unit normal derivative to $\partial \Omega$. By $Q_{\omega}$ we denote the cylinder $Q_{\omega}=(0, T) \times \omega$. Let $\xi=\left(\xi^{\prime}, \xi_{2}\right)$, $\xi^{\prime}=\left(\xi_{0}, \xi_{1}\right)$. We set

$$
\begin{aligned}
& p_{1}(x, \xi)=\rho\left(x^{\prime}\right) \xi_{0}^{2}-\mu\left(x^{\prime}\right)\left(\left|\xi_{1}\right|^{2}+\left|\xi_{2}\right|^{2}\right), \\
& p_{2}(x, \xi)=\rho\left(x^{\prime}\right) \xi_{0}^{2}-\left(\lambda\left(x^{\prime}\right)+2 \mu\left(x^{\prime}\right)\right)\left(\left|\xi_{1}\right|^{2}+\left|\xi_{2}\right|^{2}\right) .
\end{aligned}
$$

For an arbitrary smooth functions $\phi(x, \xi), \psi(x, \xi)$ we define the Poisson bracket by the formula $\{\phi, \psi\}=\sum_{i=0}^{2}\left(\frac{\partial \phi}{\partial \xi_{i}} \frac{\partial \psi}{\partial x_{i}}-\frac{\partial \phi}{\partial x_{i}} \frac{\partial \psi}{\partial \xi_{i}}\right)$. We assume that the Lamé coefficients $\mu, \lambda$ and the domains $\Omega, \omega$ satisfy the following condition

Condition 1 There exists a function $\psi \in C^{2}(\bar{Q})$ such that

$$
\begin{gathered}
\left\{p_{k},\left\{p_{k}, \psi\right\}\right\}(x, \xi)>0 \quad \forall k \in\{1,2\} \\
\forall \xi \in \mathbb{R}^{3} \backslash\{0\}, p_{k}(x, \xi)=<\frac{\partial p_{k}}{\partial \xi}, \nabla \psi>=0, x \in \overline{Q \backslash Q_{\omega}} . \\
\left\{p_{k}(x, \xi-i s \nabla \psi(x)), p_{k}(x, \xi+i s \nabla \psi(x))\right\} / 2 i s>0
\end{gathered}
$$

for all $\xi \in \mathbb{R}^{3} \backslash\{0\}, s \neq 0, p_{k}(x, \xi+i s \nabla \psi(x))=<\partial_{\xi} p_{k}(x, \xi+i s \nabla \psi(x)), \nabla \psi(x)>=$ $0, \quad x \in \overline{Q \backslash Q_{\omega}}$.

On the lateral boundary we assume

$$
\left.p_{1}(x, \nabla \psi)\right|_{(0, T) \times(\partial \Omega \backslash \partial \omega)}<0,\left.\quad \frac{\partial \psi}{\partial \vec{\nu}}\right|_{(0, T) \times(\partial \Omega \backslash \partial \omega)}<0 .
$$

Let $\psi(x)$ be the weight function from Condition 1.1. Using this function we introduce the function $\phi(x)$ by formula

$$
\phi(x)=e^{\lambda \psi(x)} \quad \lambda>1,
$$

where parameter $\lambda$ will be fixed below.

Now we formulate our main result. 
Theorem 1 Let $f \in L^{2}\left(0, T ;\left(H^{1}(\Omega)\right)^{2}\right)$, function $\phi$ is given by (5) and Lamé coefficients satisfy (3). Then there exist $\hat{\lambda}$ such that for any $\lambda>\hat{\lambda}$ exists $s_{0}$ such that for any solution $u \in\left(H_{0}^{1}(Q)\right)^{2} \cap L^{2}\left(0, T ;\left(H^{2}(\Omega)\right)^{2}\right)$ to problem (1), (2) the following estimate holds true

$$
\begin{gathered}
\int_{Q}\left(s^{2}|\nabla u|^{2}+s^{4}|u|^{2}+s|\nabla \operatorname{rot} u|^{2}+s^{3}|\operatorname{rot} u|^{2}+s|\nabla \operatorname{divu}|^{2}+s^{3}|\operatorname{div} u|^{2}\right) e^{2 s \phi} d x \\
+s\left\|\frac{\partial u}{\partial \nu} e^{s \phi}\right\|_{\left(H^{1}((0, T) \times \partial \Omega)\right)^{2}}^{2}+s\left\|\frac{\partial^{2} u}{\partial \nu^{2}} e^{s \phi}\right\|_{\left(L^{2}((0, T) \times \partial \Omega)\right)^{2}}^{2}+s^{3}\left\|\frac{\partial u}{\partial \nu} e^{s \phi}\right\|_{\left.\left(L^{2}((0, T) \times \partial \Omega)\right)\right)^{2}}^{2} \\
\leq C_{1}\left(s\left\|f e^{s \phi}\right\|_{\left(L^{2}((0, T) \times \partial \Omega)\right)^{2}}^{2}+\right. \\
\left\|\operatorname{rot} f e^{s \phi}\right\|_{\left(L^{2}(Q)\right)^{2}}^{2}+\left\|\operatorname{div} f e^{s \phi}\right\|_{\left(L^{2}(Q)\right)^{2}}^{2}+\int_{Q_{\omega}}\left(s^{2}|\nabla u|^{2}+s^{4}|u|^{2}\right) e^{2 s \phi} d x+ \\
\left.\int_{Q_{\omega}}\left(s|\nabla \operatorname{rot} u|^{2}+s^{3}|\operatorname{rot} u|^{2}+s|\nabla \operatorname{divu}|^{2}+s^{3}|\operatorname{div} u|^{2}\right) e^{2 s \phi} d x\right) \quad \forall s \geq s_{0}(\lambda),
\end{gathered}
$$

where constant $C_{1}$ is independent of $s$.

For controllability problems we need some variants of Carleman estimate (6). In addition to Condition 1.1 we assume

$$
\frac{\partial \phi\left(T, x^{\prime}\right)}{\partial x_{0}}<0, \quad \frac{\partial \phi\left(0, x^{\prime}\right)}{\partial x_{0}}>0 \quad \forall x \in \bar{\Omega} .
$$

We have

Theorem 2 Let $f \in\left(L^{2}(Q)\right)^{2}$, function $\phi$ is given by (5), satisfies (7) and Lamé coefficients satisfy (3). Then there exist $\hat{\lambda}$ such that for any $\lambda>\hat{\lambda}$ exists $s_{0}$ such that for any solution $u \in\left(H^{1}(Q)\right)^{2}$ to problem (1), (2) the following estimate holds true

$$
\begin{aligned}
& \int_{Q}\left(|\nabla u|^{2}+s^{2}|u|^{2}\right) e^{2 s \phi} d x \\
& \quad \leq C_{1}\left(\left\|f e^{s \phi}\right\|_{\left(L^{2}(Q)\right)^{2}}^{2}+\int_{Q_{\omega}}\left(|\nabla u|^{2}+s^{2}|u|^{2}\right) e^{2 s \phi} d x\right) \quad \forall s \geq s_{0}(\lambda),
\end{aligned}
$$

where constant $C_{1}$ is independent of $s$.

Corollary 1 Let $f \in L^{2}\left(0, T ;\left(H^{-1}(\Omega)\right)^{2}\right)$, function $\phi$ is given by (5) satisfies (7) and Lamé coefficients satisfy (3). Then there exist $\hat{\lambda}$ such that for any $\lambda>\hat{\lambda}$ exists $s_{0}$ such that for any solution $u \in\left(L^{2}(Q)\right)^{2}$ to problem (1), (2) the following estimate holds true

$$
\begin{array}{r}
\int_{Q}|u|^{2} e^{2 s \phi} d x \\
\quad \leq C_{1}\left(\left\|f e^{s \phi}\right\|_{L^{2}\left(0, T ;\left(H^{-1}(\Omega)\right)^{2}\right)}^{2}+\int_{Q_{\omega}}|u|^{2} e^{2 s \phi} d x\right) \quad \forall s \geq s_{0}(\lambda),
\end{array}
$$

where constant $C_{1}$ is independent of $s$. 
Now we would like to consider the applications of the Carleman estimates to the exact controllability problem of the Lamé system:

$$
\begin{aligned}
P u=f+\chi_{\omega} v \quad \text { in } Q, \quad & \left.u\right|_{(0, T) \times \partial \Omega}=0, \\
& u(0, \cdot)=u_{0}, u_{x_{0}}(0, \cdot)=u_{1}, u(T, \cdot)=u_{x_{0}}(T, \cdot)=0 .
\end{aligned}
$$

Here the functions $u_{0}, u_{1}, f$ are given and $v$ is a control locally distributed in a domain $Q_{\omega}$. The following theorem is the direct corollary of the Carleman estimates (8), (9) and the Hilbert Uniqueness Method.

Theorem 3 Let function $\psi$ satisfies the Condition 1.1 and there exists $\tau \in(0, T)$ such that

$$
\min _{x^{\prime} \in \bar{\Omega}} \psi\left(\tau, x^{\prime}\right)>\max _{x^{\prime} \in \bar{\Omega}}\left\{\psi\left(T, x^{\prime}\right), \psi\left(-T, x^{\prime}\right)\right\} .
$$

Then

A. If $u_{0} \in\left(H_{0}^{1}(\Omega)\right)^{2}, u_{1} \in\left(L^{2}(\Omega)\right)^{2}, f \in\left(L^{2}(Q)\right)^{2}$ then there exists a solution to problem (5.45) a pair $(u, v) \in\left(H^{1}(Q)\right)^{2} \times\left(L^{2}\left(Q_{\omega}\right)\right)^{2}$.

B. If $u_{0} \in\left(L^{2}(\Omega)\right)^{2}, u_{1} \in\left(H^{-1}(\Omega)\right)^{2}, f \in L^{2}\left(0, T ;\left(H^{-1}(\Omega)\right)^{2}\right)$ then there exists a solution to problem $(10)$ a pair $(u, v) \in\left(L^{2}(Q)\right)^{2} \times L^{2}\left(0, T ;\left(H^{-1}(\Omega)\right)^{2}\right)$, supp $v \subset \bar{Q}_{\omega}$.

Proof of Theorem 1.1. Withought the loss of generality we may assume $\rho \equiv 1$. First we note that instead of (6) it suffices to prove more simple estimate

$$
\begin{aligned}
& \int_{Q}\left(s|\nabla \operatorname{rot} u|^{2}+s^{3}|\operatorname{rot} u|^{2}+s|\nabla \operatorname{div} u|^{2}+s^{3}|\operatorname{div} u|^{2}\right) e^{2 s \phi} d x \\
& +s\left\|\frac{\partial u}{\partial \nu} e^{s \phi}\right\|_{\left(H^{1}((0, T) \times \partial \Omega)\right)^{2}}^{2}+s\left\|\frac{\partial^{2} u}{\partial \nu^{2}} e^{s \phi}\right\|_{\left(L^{2}((0, T) \times \partial \Omega)\right)^{2}}^{2}+s^{3}\left\|\frac{\partial u}{\partial \nu} e^{s \phi}\right\|_{\left(L^{2}((0, T) \times \partial \Omega)\right)^{2}}^{2} \\
& \quad \leq C_{2}\left(s\left\|f e^{s \phi}\right\|_{\left(L^{2}((0, T) \times \partial \Omega)\right)^{2}}^{2}+\left\|\operatorname{rot} f e^{s \phi}\right\|_{\left(L^{2}(Q)\right)^{2}}^{2}+\left\|\operatorname{div} f e^{s \phi}\right\|_{\left(L^{2}(Q)\right)^{2}}^{2}\right. \\
& \left.+\int_{Q_{\omega}}\left(s|\nabla \operatorname{rot} u|^{2}+s^{3}|\operatorname{rot} u|^{2}+s|\nabla \operatorname{div} u|^{2}+s^{3}|\operatorname{div} u|^{2}\right) e^{2 s \phi} d x\right) \quad \forall s \geq s_{0}(\hat{\lambda}) .
\end{aligned}
$$

This fact is the simple corollary of the following proposition

Proposition 1 There exists $\hat{\lambda}>1$ such that for any $\lambda>\hat{\lambda}$ exist $s_{0}(\lambda)$ that

$$
\begin{aligned}
\int_{Q}\left(|\nabla u|^{2}+s^{2}|u|^{2}\right) e^{2 s \phi} & d x \leq C_{3}\left(\left\|\operatorname{rot} u e^{s \phi}\right\|_{L^{2}(Q)}^{2}+\left\|\operatorname{div} u e^{s \phi}\right\|_{L^{2}(Q)}^{2}\right. \\
+ & \left.\int_{Q_{\omega}}\left(|\nabla u|^{2}+s^{2}|u|^{2}\right) e^{2 s \phi} d x\right) \quad \forall s \geq s_{0}(\lambda), u \in\left(H_{0}^{1}(Q)\right)^{2} .
\end{aligned}
$$

Denote by $\square_{\beta}$ the following hyperbolic operator $\square_{\beta}=\frac{\partial^{2}}{\partial x_{0}^{2}}-\beta(x) \Delta$. It is well known that the functions $\operatorname{rot} u, \operatorname{div} u$ satisfy the equations

$$
\begin{gathered}
\square_{\mu} \operatorname{rot} \mathrm{u}=q_{1} \quad \text { in } Q, \quad \square_{\lambda+2 \mu} \operatorname{div} \mathrm{u}=q_{2} \quad \text { in } Q, \\
q_{1}=K_{1} \operatorname{rot} u+K_{2} \operatorname{div} u+\operatorname{rot} f, \quad q_{2}=K_{3} \operatorname{rot} u+K_{4} \operatorname{div} u+\operatorname{div} f,
\end{gathered}
$$


where $K_{i}$ are the first order differential operators with $L^{\infty}$ coefficients.

We observe that it suffices to prove Theorem 1.1 only locally, assuming

$$
\operatorname{supp} u \subset B_{\delta}
$$

where $B_{\delta}$ is the ball of the radius $\delta>0$ centered at some point $y^{*}$. When $B_{\delta} \cap$ $(0, T) \times \partial \Omega=\emptyset$ the situation is trivial (see e.g. [ Hö]). Therefore, without the loss of generality we may assume that $y^{*}=\left(y_{0}^{*}, 0,0\right)$. Moreover the parameter $\delta>0$ could be chosen an arbitrary small. Assume that locally near zero the boundary $\partial \Omega$ is given by equation $x_{2}-\ell\left(x_{1}\right)=0$. Moreover since the function $\tilde{u}=\mathcal{O} u\left(t, \mathcal{O}^{-1} x\right)$ satisfies the system $(1),(2)$ with $\tilde{f}=\mathcal{O} f\left(t, \mathcal{O}^{-1} x\right)$ for any orthogonal matrix $\mathcal{O}$ we may assume that

$$
\ell^{\prime}(0)=0
$$

Making the change of variables $y_{1}=x_{1}, y_{2}=x_{2}-\ell\left(x_{1}\right)$ we reduce equations (1) to the form

$$
\begin{array}{r}
\frac{\partial^{2} u_{1}}{\partial y_{0}^{2}}-\mu\left(\frac{\partial^{2} u_{1}}{\partial y_{1}^{2}}-2 \ell^{\prime}\left(y_{1}\right) \frac{\partial^{2} u_{1}}{\partial y_{1} \partial y_{2}}+\left(1+\left|\ell^{\prime}\left(y_{1}\right)\right|^{2}\right) \frac{\partial^{2} u_{1}}{\partial y_{2}^{2}}\right)+\mu \ell^{\prime \prime}\left(y_{1}\right) \frac{\partial u_{1}}{\partial y_{2}} \\
-(\lambda+\mu) \frac{\partial}{\partial y_{1}}\left(\operatorname{div} u-\frac{\partial u_{1}}{\partial y_{2}} \ell^{\prime}\right)+(\lambda+\mu) \frac{\partial}{\partial y_{2}}\left(\operatorname{div} u-\frac{\partial u_{1}}{\partial y_{2}} \ell^{\prime}\right) \ell^{\prime}=f_{1}, \\
\frac{\partial^{2} u_{2}}{\partial y_{0}^{2}}-\mu\left(\frac{\partial^{2} u_{2}}{\partial y_{1}^{2}}-2 \ell^{\prime}\left(y_{1}\right) \frac{\partial^{2} u_{2}}{\partial y_{1} \partial y_{2}}+\left(1+\left|\ell^{\prime}\left(y_{1}\right)\right|^{2}\right) \frac{\partial^{2} u_{2}}{\partial y_{2}^{2}}\right)+\mu \ell^{\prime \prime}\left(y_{1}\right) \frac{\partial u_{2}}{\partial y_{2}} \\
-(\lambda+\mu) \frac{\partial}{\partial y_{2}}\left(\operatorname{div} u-\frac{\partial u_{1}}{\partial y_{2}} \ell^{\prime}\right)=f_{2},
\end{array}
$$

where by $f_{1}, f_{2}$ we denote $f$ after the change of variables. After the change of variables equations (12), (13) have the form

$$
\begin{gathered}
P_{\mu} z_{1}=\frac{\partial^{2} z_{1}}{\partial y_{0}^{2}}-\mu\left(\frac{\partial^{2} z_{1}}{\partial y_{1}^{2}}-2 \ell^{\prime}\left(y_{1}\right) \frac{\partial^{2} z_{1}}{\partial y_{1} \partial y_{2}}+\left(1+\left|\ell^{\prime}\left(y_{1}\right)\right|^{2}\right) \frac{\partial^{2} z_{1}}{\partial y_{2}^{2}}\right) \\
+\mu \ell^{\prime \prime}\left(y_{1}\right) \frac{\partial z_{1}}{\partial y_{2}}=m_{1} \quad \text { in } \mathcal{G} \triangleq \mathbb{R}^{2} \times[0, \hat{\kappa}] \\
P_{\lambda+2 \mu} z_{2}=\frac{\partial^{2} z_{2}}{\partial y_{0}^{2}}-(\lambda+2 \mu)\left(\frac{\partial^{2} z_{2}}{\partial y_{1}^{2}}-2 \ell^{\prime}\left(y_{1}\right) \frac{\partial^{2} z_{2}}{\partial y_{1} \partial y_{2}}+\left(1+\left|\ell^{\prime}\left(y_{1}\right)\right|^{2}\right) \frac{\partial^{2} z_{2}}{\partial y_{2}^{2}}\right) \\
+(\lambda+2 \mu) \ell^{\prime \prime}\left(y_{1}\right) \frac{\partial z_{2}}{\partial y_{2}}=m_{2} \quad \text { in } \mathcal{G},
\end{gathered}
$$

and $m_{1}, m_{2}$ are the functions $q_{1}, q_{2}$ after the change of variables.

Without the loss of the generality we may assume $\hat{\kappa}=1$. Next we introduce the operators

$$
P_{\mu, s}=e^{|s| \phi} P_{\mu} e^{-|s| \phi}, \quad P_{\lambda+2 \mu, s}=e^{|s| \phi} P_{\lambda+2 \mu} e^{-|s| \phi} .
$$


Now we note that in order to prove estimate (11) it suffices to establish the following estimate for the function $w=\left(w_{1}, w_{2}\right)$ :

$$
\begin{aligned}
&\|w\|_{*}^{2} \equiv s\|w\|_{\left(H^{1}(\mathcal{G})\right)^{2}}^{2}+s^{3}\|w\|_{\left(L^{2}(\mathcal{G})\right)^{2}}^{2}+s\left\|\frac{\partial w}{\partial y_{2}}\right\|_{\left(L^{2}(\partial \mathcal{G})\right)^{2}}^{2}+\mid w \|_{\left(H^{1}(\partial \mathcal{G})\right)^{2}}^{2} \\
&+s^{3}\left\|\frac{\partial w}{\partial y_{2}}\right\|_{\left(L^{2}(\partial \mathcal{G})\right)^{2}}^{2} \leq C_{4}\left(\left\|P_{\lambda+2 \mu, s} w_{2}\right\|_{L^{2}(\mathcal{G})}^{2}+\left\|P_{\mu, s} w_{1}\right\|_{L^{2}(\mathcal{G})}^{2}+s\|g\|_{\left(L^{2}(\partial \mathcal{G})\right)^{2}}^{2}\right. \\
&\left.+s\|w\|_{\left(H^{1}\left(\mathcal{G}_{\omega}\right)\right)^{2}}^{2}+s^{3}\|w\|_{\left(L^{2}\left(\mathcal{G}_{\omega}\right)\right)^{2}}^{2}\right) \quad \forall s \geq s_{0}(\lambda),
\end{aligned}
$$

and for all $w \in H^{2}(\mathcal{G}), w(\cdot, T)=w(\cdot, T)=w_{y_{0}}(\cdot, 0)=w_{y_{0}}(\cdot, T)=0$ such that

$$
\begin{array}{cc}
\frac{\partial w_{1}}{\partial y_{2}}=\frac{\lambda+2 \mu}{\mu} \frac{\partial w_{2}}{\partial y_{1}}+s \phi_{y_{2}}\left(y^{*}\right) w_{1}-s \frac{\lambda+2 \mu}{\mu} \phi_{y_{1}}\left(y^{*}\right) w_{2}+g_{1}, \quad \text { on } \partial \mathcal{G}, \\
\frac{\partial w_{2}}{\partial y_{2}}=-\frac{\mu}{\lambda+2 \mu} \frac{\partial w_{1}}{\partial y_{1}}+s \phi_{y_{2}}\left(y^{*}\right) w_{2}+s \frac{\mu}{\lambda+2 \mu} \phi_{y_{1}}\left(y^{*}\right) w_{1}+g_{2}, \quad \text { on } \partial \mathcal{G} .
\end{array}
$$

We denote as $p_{\mu}\left(y, \xi_{0}, \xi_{1}, \xi_{2}\right), p_{\lambda+2 \mu}\left(y, \xi_{0}, \xi_{1}, \xi_{2}\right)$ respectively the principal symbols of the operators $P_{\mu}, P_{\lambda+2 \mu}$. Then the principal symbols of the operators $P_{s, \mu}, P_{s, \lambda+2 \mu}$ principal are given by formulas

$$
\begin{aligned}
& p_{\mu}\left(y, \xi_{0}+i|s| \phi_{y_{0}}, \xi_{1}+i|s| \phi_{y_{1}}, \xi_{2}+i|s| \phi_{y_{2}}\right), \\
& p_{\lambda+2 \mu}\left(y, \xi_{0}+i|s| \phi_{y_{0}}, \xi_{1}+i|s| \phi_{y_{1}}, \xi_{2}+i|s| \phi_{y_{2}}\right) .
\end{aligned}
$$

In order to prove the Carleman estimate it is convenient for us to introduce new variable $\sigma$ and consider $s$ as a dual variable to $\sigma$. Following [T1, Chapter 14] we consider the pseudodifferential operators

$$
\begin{aligned}
& \mathbf{P}_{\beta}\left(y, D_{\sigma}, D_{y_{0}}, D_{y_{1}}, D_{y_{2}}\right) v= \\
& \quad \int_{\mathbb{R}^{3}} p_{\beta}\left(y, \xi_{0}+i|s| \phi_{y_{0}}, \xi_{1}+i|s| \phi_{y_{1}}, D_{y_{2}}+i|s| \phi_{y_{2}}\right) \hat{v}\left(s, \xi_{0}, \xi_{1}, y_{2}\right) e^{\left.i\left(<y^{\prime}, \xi^{\prime}\right\rangle+\sigma s\right)} d \sigma d \xi^{\prime},
\end{aligned}
$$

where $\xi^{\prime}=\left(\xi_{0}, \xi_{1}\right), y^{\prime}=\left(y_{0}, y_{1}\right)$. Let $\mathbf{v}(\sigma, y)=\left(v_{1}(\sigma, y), v_{2}(\sigma, y)\right)$ be the function with the domain $\mathcal{Q}=\mathbb{R}^{2} \times \mathbb{R}_{+}^{1} \times \mathbb{R}^{1}(\Sigma=\partial \mathcal{Q}$. $)$ and $w(s, y)=\left(w_{1}(s, y), w_{2}(s, y)\right)$ be the Fourier transform of $\mathbf{v}$ respect to the variable $\sigma$. Let $h(s)=\left(1+s^{2}\right)^{\frac{1}{4}}$. Using the method developed in [T1] we obtain that in order to prove (11) it suffices to establish the following estimate

$$
\begin{gathered}
\|\| \mathbf{v}\left\|\left.\right|^{2} \triangleq \sum_{j=0}^{1}\right\| h\left(D_{\sigma}\right)^{3-2 j} \mathbf{v}\left\|_{L^{2}\left(\mathbb{R}^{1} ;\left(H^{j}(\mathcal{G})\right)^{2}\right)}^{2}+\right\| h\left(D_{\sigma}\right)^{3-2 j} \mathbf{v}\left\|_{\left(H^{j}( \pm)\right)^{2}}^{2}+\right\| h\left(D_{\sigma}\right) \frac{\partial \mathbf{v}}{\partial y_{2}} \|_{\left(L^{2}(\Sigma)\right)^{2}} \\
\leq C_{10}\left(\left\|\mathbf{P}_{\lambda+2 \mu}(y, D) v_{1}\right\|_{L^{2}(\mathcal{Q})}^{2}+\left\|\mathbf{P}_{\mu}(y, D) v_{2}\right\|_{L^{2}(\mathcal{Q})}^{2}\right. \\
\left.+\left\|h\left(D_{\sigma}\right) q\right\|_{\left(L^{2}(\Sigma)\right)^{2}}^{2}+\|\mathbf{v}\|_{\left(H^{1}(\mathcal{Q})\right)^{2}}^{2}\right),
\end{gathered}
$$

where $g=\frac{1}{\sqrt{2 \pi}} \int_{-\infty}^{+\infty} q e^{-i s \sigma} d \sigma, \operatorname{supp} \mathbf{v} \subset\left(-\sigma_{0}, \sigma_{0}\right) \times B_{\delta}$ with the parameter $\sigma_{0}>0$ which can be chosen an arbitrary small, and

$B_{1}\left(w_{1}, w_{2}\right) \triangleq-\frac{\partial w_{1}}{\partial y_{2}}+\frac{\lambda+2 \mu}{\mu} \frac{\partial w_{2}}{\partial y_{1}}+|s| \phi_{y_{2}}\left(y^{*}\right) w_{1}-|s| \frac{\lambda+2 \mu}{\mu} \phi_{y_{1}}\left(y^{*}\right) w_{2}=g_{1}, \quad$ on $\Sigma$, 
$B_{2}\left(w_{1}, w_{2}\right) \triangleq-\frac{\partial w_{2}}{\partial y_{2}}-\frac{\mu}{\lambda+2 \mu} \frac{\partial w_{1}}{\partial y_{1}}+|s| \phi_{y_{2}}\left(y^{*}\right) w_{2}+|s| \frac{\mu}{\lambda+2 \mu} \phi_{y_{1}}\left(y^{*}\right) w_{1}=g_{2}, \quad$ on $\Sigma$,

where

$$
w=\frac{1}{\sqrt{2 \pi}} \int_{-\infty}^{+\infty} \mathbf{v} e^{-i s \sigma} d \sigma
$$

This fact can be proved in exactly the same way as in [T1, Chapter 14 ,section 2].

Consider the finite covering of the unit sphere $s^{2}+\xi_{0}^{2}+\xi_{1}^{2}=1: S^{2} \subset \cup_{\zeta^{*} \in S^{2}}\{\zeta=$ $\left.\left(s, \xi_{0}, \xi_{1}\right) \in S^{2}|| \zeta-\zeta^{*} \mid<\delta_{1}\right\}$ and submitted to this covering partition of unity $\chi_{\nu}(\zeta)$ : $\sum_{\nu=1}^{N} \chi_{\nu}(\zeta)=1$ for any $\zeta \in S^{2}$ and supp $\chi_{\nu} \subset\left\{\zeta \in S^{2}|| \zeta-\zeta_{\nu}^{*} \mid<\delta_{1}\right\}$. We extend this function on the set $|\zeta|>1$ as the homogeneous function of the order zero in a such a way that $\operatorname{supp}_{\nu} \subset \mathcal{O}\left(\delta_{1}\right)=\left\{\zeta|| \frac{\zeta}{|\zeta|}-\zeta^{*} \mid<\delta_{1}\right\}$. Let us consider the pseudodifferential operator $\chi_{\nu}(D)$ and the function $\chi_{\nu}(D) \mathbf{v}$. Obviously equalities $(16),(17)$ holds true with $w_{\nu}=\frac{1}{\sqrt{2 \pi}} \int_{-\infty}^{+\infty} \chi_{\nu}(D) \mathbf{v} e^{-i s \sigma} d \sigma, g_{\nu}=\frac{1}{\sqrt{2 \pi}} \int_{-\infty}^{+\infty} \chi_{\nu}(D) q e^{-i s \sigma} d \sigma$.

We claim that instead of (15) it suffices to prove the following estimate

$$
\begin{aligned}
\left\|\chi_{\nu}(D) \mathbf{v}\right\| \leq C_{10}(\nu)\left(\left\|\mathbf{P}_{\lambda+2 \mu} \chi_{\nu}(D) \mathbf{v}\right\|_{L^{2}(\mathcal{Q})}+\left\|\mathbf{P}_{\mu} \chi_{\nu}(D) \mathbf{v}\right\|_{L^{2}(\mathcal{Q})}\right. \\
\left.+\left\|h\left(D_{\sigma}\right) \chi_{\nu}(D) q\right\|_{L^{2}(\Sigma)}+\|\mathbf{v}\|_{\left(H^{1}(\mathcal{Q})\right)^{2}}\right),
\end{aligned}
$$

where

$$
B_{1}\left(w_{1, \nu}, w_{2, \nu}\right)=g_{1, \nu}, \quad B_{2}\left(w_{1, \nu}, w_{2, \nu}\right)=g_{2, \nu} .
$$

The principal symbol of the operator $P_{\beta, s}$ has the form

$$
\begin{array}{r}
p\left(y, s, \xi_{0}, \xi_{1}\right)=-\left(\xi_{0}+i|s| \phi_{y_{0}}\right)^{2}+\beta\left[\left(\xi_{1}+i|s| \phi_{y_{1}}\right)^{2}-2 \ell^{\prime}\left(\xi_{1}+i|s| \phi_{y_{1}}\right)\left(\xi_{2}+i|s| \phi_{y_{2}}\right)\right. \\
\left.+\left(\xi_{2}+i|s| \phi_{y_{2}}\right)^{2}|G|\right], \quad(20)
\end{array}
$$

where $|G|=1+\left(\ell^{\prime}\right)^{2}$. The roots of this polynomial respect to variable $\xi_{2}$ are

$$
\begin{gathered}
\Gamma_{\beta}^{ \pm}\left(y, s, \xi_{0}, \xi_{1}\right)=-i|s| \phi_{y_{2}}+\alpha_{\beta}^{ \pm}\left(y, s, \xi_{0}, \xi_{1}\right), \\
\alpha_{\beta}^{ \pm}\left(y, s, \xi_{0}, \xi_{1}\right)=\frac{\left(\xi_{1}+i|s| \phi_{y_{1}}\right) \ell^{\prime}\left(y_{1}\right)}{|G|} \pm \sqrt{r_{\beta}\left(y, s, \xi_{0}, \xi_{1}\right)}, \\
r_{\beta}(y, \zeta)=\frac{\left(\left(\xi_{0}+i|s| \phi_{y_{0}}\right)^{2}-\beta\left(\xi_{1}+i|s| \phi_{y_{1}}\right)^{2}\right)|G|+\beta\left(\xi_{1}+i|s| \phi_{y_{1}}\right)^{2}\left(\ell^{\prime}\right)^{2}}{\beta|G|^{2}} .
\end{gathered}
$$

Denote $\gamma=\left(y^{*}, \zeta^{*}\right)$. Suppose that $r_{\beta}(\gamma) \neq 0$. Now we claim that there exists $\delta_{0}>0$ such that for all $\delta, \delta_{1} \in\left(0, \delta_{0}\right)$ there exists a constant $\hat{C}_{14}>0$ such that for one of the roots of the polynomial (20), which we denote as $\Gamma_{\beta}^{-}$we have

$$
-\operatorname{Im} \Gamma_{\beta}^{-}\left(y, s, \xi_{0}, \xi_{1}\right) \geq \hat{C}_{14}|s| \quad \forall\left(y, s, \xi_{0}, \xi_{1}\right) \in B_{\delta} \times \mathcal{O}\left(\delta_{1}\right) .
$$

In some situations we may represent the operator $\mathbf{P}_{\beta}$ as a product of two first order pseudodifferential operators. 
Proposition 2 Let $r_{\beta}(\gamma) \neq 0$ and supp $\hat{V} \subset \mathcal{O}\left(\delta_{1}\right)$. Then we could decompose the operator $P_{s, \beta}$ into the product of two first order P.D.O.

$$
\mathbf{P}_{\beta} V=\left(D_{y_{2}}-\Gamma_{\beta}^{-}(y, D)\right)\left(D_{y_{2}}-\Gamma_{\beta}^{+}(y, D)\right) V+T_{\beta} V
$$

where $T_{\beta}$ is the continuous operator

$$
T_{\beta}: L^{2}\left(0,1 ; H^{1}\left(\mathbb{R}^{3}\right)\right) \rightarrow L^{2}\left(0,1 ; L^{2}\left(\mathbb{R}^{3}\right)\right) .
$$

Let us consider the equation

$$
\left(D_{y_{2}}-\Gamma_{\beta}^{-}(y, D)\right) V=q,\left.\quad V\right|_{y_{2}=1}=0 .
$$

For solutions of this problem we have the a priori estimate:

Proposition 3 There exists a constant $C_{15}>0$ such that

$$
\left\|\left.h\left(D_{\sigma}\right) V\right|_{y_{2}=0}\right\|_{L^{2}\left(\mathbb{R}^{3}\right)} \leq C_{15}\|q\|_{L^{2}(\mathcal{Q})} .
$$

Let $\beta>0$ and $\tilde{w}(s, y)$ be a function which for almost all $s \in \mathbb{R}^{1}$ satisfies the scalar second order hyperbolic equation

$$
P_{\beta, s} \tilde{w}=q \quad \text { in } \mathcal{G},\left.\quad \frac{\partial \tilde{w}}{\partial y_{2}}\right|_{y_{2}=1}=\left.\tilde{w}\right|_{y_{2}=1}=0, \quad \operatorname{supp} \tilde{w} \subset B_{\delta} \times \mathbb{R}^{1} .
$$

Let $P_{\beta, s}^{*}$ be the formally adjoint operator to $P_{s, \beta}$, where $\beta \in[\mu, \lambda+2 \mu]$. Set $L_{+, \beta}=$ $\left(P_{\beta, s}+P_{\beta, s}^{*}\right) / 2$ and $L_{-, \beta}=\left(P_{\beta, s}-P_{\beta, s}^{*}\right) / 2$. One may easily check that the operator $L_{-, \beta}$ is given by the formula

$$
\begin{aligned}
L_{-, \beta} \tilde{w} & =-2|s| \phi_{y_{0}} \frac{\partial \tilde{w}}{\partial y_{0}} \\
& +\beta\left(2|s| \phi_{y_{1}} \frac{\partial \tilde{w}}{\partial y_{1}}-2|s| \ell^{\prime}\left(y_{1}\right)\left(\phi_{y_{2}} \frac{\partial \tilde{w}}{\partial y_{1}}+\phi_{y_{1}} \frac{\partial \tilde{w}}{\partial y_{2}}\right)+2|s|\left(1+\ell^{\prime}\left(y_{1}\right)^{2}\right) \phi_{y_{2}} \frac{\partial \tilde{w}}{\partial y_{2}}\right) .
\end{aligned}
$$

Obviously $L_{+, \beta} \tilde{w}+L_{-, \beta} \tilde{w}=q$. For almost all $s \in \mathbb{R}^{1}$ the following equality holds true

$$
B_{\beta}+\left\|L_{-, \beta} \tilde{w}\right\|_{L^{2}(\mathcal{G})}^{2}+\left\|L_{+, \beta} \tilde{w}\right\|_{L^{2}(\mathcal{G})}^{2}+\operatorname{Re}\left(\left[L_{+, \beta}, L_{-, \beta}\right] \tilde{w}, \tilde{w}\right)_{L^{2}(\mathcal{G})}=\|q\|_{L^{2}(\mathcal{G})}^{2},
$$

where

$$
\begin{array}{r}
B_{\beta}=\operatorname{Re} \int_{\partial \mathcal{G}} \tilde{p}_{\beta}\left(y, \nabla \phi,-\vec{e}_{2}\right)\left(|s| \tilde{p}_{\beta}(y, \nabla \tilde{w})-|s|^{3} \tilde{p}_{\beta}(y, \nabla \phi, \nabla \phi) \tilde{w}^{2}\right) d y_{0} d y_{1} \\
+\operatorname{Re} \int_{\partial \mathcal{G}} \tilde{p}_{\beta}\left(y, \nabla \tilde{w},-\vec{e}_{2}\right) \overline{L_{-, \beta} \tilde{w}} d y_{0} d y_{1},
\end{array}
$$

$\vec{e}=(0,0,1)$ and

$$
\tilde{p}_{\beta}(y, \xi, \tilde{\xi})=\xi_{0} \tilde{\xi}_{0}-\beta\left(\xi_{1} \tilde{\xi}_{1}-\ell^{\prime}\left(y_{1}\right)\left(\xi_{1} \tilde{\xi}_{2}+\xi_{2} \tilde{\xi}_{1}\right)+\left(1+\left|\ell^{\prime}\left(y_{1}\right)\right|^{2}\right) \xi_{2} \tilde{\xi}_{2}\right) .
$$


It is convenient for us to rewrite $(25)$ in the form

$$
\begin{gathered}
B_{\beta}=B_{\beta}^{(1)}+B_{\beta}^{(2)} \\
B_{\beta}^{(1)}=\operatorname{Re} \int_{y_{2}=0} 2|s| \beta \frac{\partial \tilde{w}}{\partial y_{2}} \frac{\left.\partial \tilde{w} \frac{\tilde{w}}{\partial y_{1}} \phi_{y_{1}}\left(y^{*}\right)+\beta \frac{\partial \tilde{w}}{\partial y_{2}} \phi_{y_{2}}\left(y^{*}\right)-\frac{\partial \tilde{w}}{\partial y_{0}} \phi_{y_{0}}\left(y^{*}\right)\right)}{d} d y_{0} d y_{1} \\
+\int_{y_{2}=0}|s| \beta \phi_{y_{2}}\left(y^{*}\right)\left(\left|\frac{\partial \tilde{w}}{\partial y_{0}}\right|^{2}-\beta\left(\left|\frac{\partial \tilde{w}}{\partial y_{1}}\right|^{2}+\left|\frac{\partial \tilde{w}}{\partial y_{2}}\right|^{2}\right)\right. \\
-|s|^{2}\left(\phi_{y_{0}}^{2}\left(y^{*}\right)-\beta\left(\phi_{y_{1}}^{2}\left(y^{*}\right)+\phi_{y_{2}}^{2}\left(y^{*}\right)\right)|\tilde{w}|^{2}\right) d y_{0} d y_{1} .
\end{gathered}
$$

and

$$
\left|B_{\beta}^{(2)}\right| \leq \epsilon_{0}\left(s\left\|\frac{\partial \tilde{w}}{\partial y_{2}}\right\|_{L^{2}(\partial \mathcal{G})}^{2}+|s|\|\tilde{w}\|_{H^{1}(\partial \mathcal{G})}^{2}+|s|^{3}\|\tilde{w}\|_{L^{2}(\partial \mathcal{G})}^{2}\right) .
$$

It is known (see e.g. [Im]) that there exists a parameter $\hat{\lambda}>1$ such that for any $\lambda>\hat{\lambda}$ there exists $s_{0}(\lambda)$ such that

$$
\begin{aligned}
\left\|L_{-, \beta} \tilde{w}\right\|_{L^{2}(\mathcal{G})}^{2}+\left\|L_{+, \beta} \tilde{w}\right\|_{L^{2}(\mathcal{G})}^{2}+ & \operatorname{Re}\left(\left[L_{+, \beta}, L_{-, \beta}\right] \tilde{w}, \tilde{w}\right)_{L^{2}(\mathcal{G})} \geq \\
& C_{18}\left(s\|\tilde{w}\|_{H^{1}(\mathcal{G})}^{2}+|s|^{3}\|\tilde{w}\|_{L^{2}(\mathcal{G})}^{2}\right) \quad \forall s \geq s_{0}(\lambda),
\end{aligned}
$$

where $C_{18}>0$ is independent of $s$. Denote by $\Xi_{\beta}=\int_{-\infty}^{\infty} B_{\beta} d s, \Xi_{\beta}^{(j)}=\int_{-\infty}^{\infty} B_{\beta}^{(j)} d s$ $j=1,2$. Therefore integrating (27) respect to $s$ on $\mathbb{R}^{1}$ we have

$$
C_{19}\left(\|h(s) \tilde{w}\|_{H^{1}(\mathcal{Q})}^{2}+\left\|h^{3}(s) \tilde{w}\right\|_{L^{2}(\mathcal{Q})}^{2}\right)+\Xi_{\beta} \leq\|q\|_{L^{2}(\mathcal{Q})}^{2}+C_{20}\|\tilde{w}\|_{H^{1}(\mathcal{Q})}^{2},
$$

where $C_{19}>0$, and by (26)

$$
\left|\Xi_{\beta}^{(2)}\right| \leq \epsilon\left\|\left(\frac{\partial \tilde{w}}{\partial y_{2}}, \tilde{w}\right)\right\|_{X}^{2}
$$

where

$$
\left\|\left(\frac{\partial \tilde{w}}{\partial y_{2}}, \tilde{w}\right)\right\|_{X}^{2}=\left\|h(s) \frac{\partial \tilde{w}}{\partial y_{2}}\right\|_{L^{2}(\Sigma)}^{2}+\|h(s) \tilde{w}\|_{L^{2}\left(\mathbb{R}^{1} ; H^{1}\left(\mathbb{R}^{2}\right)\right)}^{2}+\|h(s) \tilde{w}\|_{L^{2}(\Sigma)}^{2}
$$

and the parameter $\epsilon$ could make an arbitrary small taking $\delta$ in (14) sufficiently small.

Later we will need to apply (28), (29) to the functions $w_{1, \nu}=F_{\sigma} \chi_{\nu}(D) v_{1}, w_{2, \nu}=$ $F_{\sigma} \chi_{\nu}(D) v_{2, \nu}$ since we would like to take the advantage of (18). Formally it is impossible since the condition $\operatorname{supp} \chi_{\nu}(D) \mathbf{v} \subset B_{\delta} \times \mathbb{R}^{1}$ in general does not hold true. On the other hand using the fact that

$$
\int_{\mathbb{R}^{2} \backslash B_{2 \delta}} \int_{\mathbb{R}^{1}} h^{4}(s) \sum_{|\alpha| \leq 2}\left|D^{\alpha} w_{i, \nu}\right|^{2} d y_{0} d y_{1} d s \leq C_{21}\|\mathbf{v}\|_{\left(H^{1}(\mathcal{Q})\right)^{2}}^{2} .
$$

we obtain slightly modified analog of (28), (29):

$$
C_{22}\left(\left\|h(s) w_{i, \nu}\right\|_{H^{1}(\mathcal{Q})}^{2}+\left\|h^{3}(s) w_{i, \nu}\right\|_{L^{2}(\mathcal{Q})}^{2}\right)+\Xi_{\beta} \leq\left\|P_{\beta, s} w_{1, \nu}\right\|_{L^{2}(\mathcal{Q})}^{2}+C_{23}\|\mathbf{v}\|_{\left(H^{1}(\mathcal{Q})\right)^{2}}^{2},
$$

where $C_{22}>0$, and $\beta=\mu$ for $i=1, \beta=\lambda+2 \mu$ for $i=2$

$$
\left|\Xi_{\beta}^{(2)}\right| \leq \epsilon\left\|\left(\frac{\partial w_{i, \nu}}{\partial y_{2}}, w_{i, \nu}\right)\right\|_{X}^{2}+C_{23}\|\mathbf{v}\|_{\left(H^{1}(\mathcal{Q})\right)^{2}}^{2} .
$$




\section{Case $r_{\mu} \neq 0$ and $r_{\lambda+2 \mu} \neq 0$.}

In this section we consider the conic neighborhood $\mathcal{O}\left(\delta_{1}\right)$ of the point $\left(y^{*}, \zeta^{*}\right)$ such that

$$
\left|r_{\mu}\left(y^{*}, \zeta^{*}\right)\right| \neq 0 \text { and }\left|r_{\lambda+2 \mu}\left(y^{*}, \zeta^{*}\right)\right| \neq 0
$$

In that case thanks to (32) and Proposition 1.2 decomposition (24) holds true for $\beta=\mu$ and $\beta=\lambda+2 \mu$. Therefore we have

$$
\begin{aligned}
\left.\left(D_{y_{2}}-\Gamma_{\mu}^{+}(y, D)\right) v_{1, \nu}\right|_{y_{2}=0} & =V_{\mu}^{+}(\cdot, 0), \\
\left.\left(D_{y_{2}}-\Gamma_{\lambda+2 \mu}^{+}(y, D)\right) v_{2, \nu}\right|_{y_{2}=0} & =V_{\lambda+2 \mu}^{+}(\cdot, 0) .
\end{aligned}
$$

By Proposition 1.3 we have the a priori estimate

$$
\begin{aligned}
\left\|h\left(D_{\sigma}\right) V_{\mu}^{+}(\cdot, 0)\right\|_{L^{2}(\Sigma)}^{2}+ & \left\|h\left(D_{\sigma}\right) V_{\lambda+2 \mu}^{+}(\cdot, 0)\right\|_{L^{2}(\Sigma)}^{2} \\
& \leq C_{1}\left(\left\|\mathbf{P}_{\lambda+2 \mu} v_{2}\right\|_{L^{2}(\mathcal{Q})}^{2}+\left\|\mathbf{P}_{\mu} v_{1}\right\|_{L^{2}(\mathcal{Q})}^{2}+\|\mathbf{v}\|_{\left(H^{1}(\mathcal{Q})\right)^{2}}^{2}\right) .
\end{aligned}
$$

Using (19) we may rewrite (33), (34) as

$$
\begin{gathered}
\frac{\lambda+2 \mu}{\mu}\left(\frac{\partial v_{2, \nu}}{\partial y_{1}}-\left|D_{\sigma}\right| \phi_{y_{1}}\left(y^{*}\right) v_{2, \nu}\right)-i \alpha_{\mu}^{+}(y, D) v_{1, \nu}=V_{\mu}^{+}(\cdot, 0)-q_{1, \nu} \\
\frac{\mu}{\lambda+2 \mu}\left(-\frac{\partial v_{1, \nu}}{\partial y_{1}}+\left|D_{\sigma}\right| \phi_{y_{1}}\left(y^{*}\right) v_{1, \nu}\right)-i \alpha_{\lambda+2 \mu}^{+}(y, D) v_{2, \nu}=V_{\lambda+2 \mu}^{+}(\cdot, 0)-q_{2, \nu}
\end{gathered}
$$

Let $\mathbf{B}(y, D)$ be the matrix P.D.O. with the symbol

$$
\mathbf{B}(y, \zeta)=\left(\begin{array}{cc}
-i \alpha_{\mu}^{+}(y, \zeta) & \frac{\lambda+2 \mu}{\mu}\left(i \xi_{1}-|s| \phi_{y_{1}}\right) \\
\frac{\mu}{\lambda+2 \mu}\left(-i \xi_{1}+|s| \phi_{y_{1}}\right) & -i \alpha_{\lambda+2 \mu}^{+}(y, \zeta)
\end{array}\right)
$$

By (22), (23) the set $\left\{\zeta^{*} \in S^{2} \mid \operatorname{det} \mathbf{B}\left(y^{*}, \zeta^{*}\right)=0\right\}$ is empty.

Then there exists a parametrix of the operator $\mathbf{B}(y, D)$ (see [T2]) which we denote as $\mathbf{B}^{-1}(y, D)$ such that

$$
\left(v_{1, \nu}, v_{2, \nu}\right)=\mathbf{B}^{-1}(y, D)\left(V_{\mu}^{+}(\cdot, 0)-q_{1, \nu}, V_{\lambda+2 \mu}^{+}(\cdot, 0)-q_{2, \nu}\right)+K\left(v_{1, \nu}, v_{2, \nu}\right),
$$

where

$$
K:\left(L^{2}(\mathcal{Q})\right) \rightarrow\left(H^{1}(\mathcal{Q})\right)^{2},
$$

By (35), (36)

$$
\left|\Xi_{\mu}\right|+\left|\Xi_{\lambda+2 \mu}\right| \leq C_{2}\left(\left\|\mathbf{P}_{\mu} v_{1}\right\|_{L^{2}(\mathcal{Q})}^{2}+\left\|\mathbf{P}_{\lambda+2 \mu} v_{2}\right\|_{L^{2}(\mathcal{Q})}^{2}+\|h(s) g\|_{\left(L^{2}(\Sigma)\right)^{2}}^{2}+\|\mathbf{v}\|_{\left(H^{1}(\mathcal{Q})\right)^{2}}^{2}\right) .
$$

By (37), (28), (29) we obtain (18). 


\section{Case $r_{\mu}=0$.}

In this section mainly we treat the case when $\operatorname{supp} \chi_{\nu} \subset \mathcal{O}\left(\delta_{1}\right)$, and $\gamma=\left(y^{*}, \zeta^{*}\right)$ be a point on $\Sigma \times S^{2}$ such that $r_{\mu}(\gamma)=0$.

We remind that by (30), (31) there exists $C_{1}>0, C_{2}>0$ such that $C_{1}\left(\left\|h(s) w_{1, \nu}\right\|_{H^{1}(\mathcal{Q})}^{2}+\left\|h^{3}(s) w_{1, \nu}\right\|_{L^{2}(\mathcal{Q})}^{2}\right)+\Xi_{\mu}^{(1)} \leq C_{2}\left\|\mathbf{P}_{\mu} v_{1}\right\|_{L^{2}(\mathcal{Q})}^{2}+\epsilon(\delta)\left\|\left(\frac{\partial w_{1, \nu}}{\partial y_{2}}, w_{1, \nu}\right)\right\|_{X}^{2}$,

and the parameter $\epsilon$ could be taken sufficiently small, if we decrease $\delta$. Note that $\Xi_{\mu}^{(1)}$ could be written in the form

$$
\begin{aligned}
\Xi_{\mu}^{(1)}=\int_{\Sigma}\left(|s| \mu^{2} \phi_{y_{2}}\left(y^{*}\right)\left|\frac{\partial w_{1, \nu}}{\partial y_{2}}\right|^{2}+|s|^{3} \mu^{2} \phi_{y_{2}}^{3}\left(y^{*}\right)\left|w_{1, \nu}\right|^{2}\right) d \Sigma \\
\quad+\operatorname{Re} \int_{\Sigma} 2|s| \mu \frac{\partial w_{1, \nu}}{\partial y_{2}} \overline{\left(\mu \phi_{y_{1}}\left(y^{*}\right) \frac{\partial w_{1, \nu}}{\partial y_{1}}-\phi_{y_{0}}\left(y^{*}\right) \frac{\partial w_{1, \nu}}{\partial y_{0}}\right)} d \Sigma \\
+\int_{\Sigma}|s| \mu \phi_{y_{2}}\left(y^{*}\right)\left(\xi_{0}^{2}-\mu \xi_{1}^{2}-s^{2} \phi_{y_{0}}^{2}\left(y^{*}\right)+s^{2} \mu \phi_{y_{1}}^{2}\left(y^{*}\right)\right)\left|\hat{v}_{1, \nu}\right|^{2} d \Sigma= \\
J_{1}+J_{2}+J_{3} .
\end{aligned}
$$

We shall consider the two cases. Let us introduce the set $\mathcal{M}$ by formula

$$
\begin{aligned}
& \mathcal{M}=\left\{\zeta=\left(s, \xi_{0}, \xi_{1}\right) \in \mathcal{O}\left(\delta_{1}\right) \mid \frac{\mu}{2} \phi_{y_{2}}\left(y^{*}\right) \hat{C} s^{2}>\right. \\
&\left.4 \mu^{2} \frac{\phi_{y_{1}}^{2}\left(y^{*}\right)}{\left|\phi_{y_{2}}\left(y^{*}\right)\right|} \xi_{1}^{2}+4 \frac{\phi_{y_{0}}^{2}\left(y^{*}\right)}{\left|\phi_{y_{2}}\left(y^{*}\right)\right|} \xi_{0}^{2}+\left(\left|\xi_{0}\right|^{2}+\left|\xi_{1}\right|^{2}\right)\right\},
\end{aligned}
$$

where $\hat{C}=\min _{y \in B_{\delta}}\left\{-p_{1}(y, \nabla \phi)\right\}$. From (4) it follows that $\hat{C}$ is positive.

Case A. Assume that

$$
\operatorname{supp} \hat{\mathbf{v}}_{\nu} \subset \mathcal{O}\left(\delta_{1}\right) \subset \mathcal{M} \text {. }
$$

Applying the Cauchy-Bunyakovskii inequality and using (40) and (4) we obtain that there exists a constant $C_{3}>0$ such that

$$
\begin{aligned}
& \Xi_{\mu}^{(1)} \geq \int_{\Sigma}|s| \mu^{2} \phi_{y_{2}}\left(y^{*}\right)\left|\frac{\partial w_{1, \nu}}{\partial y_{2}}\right|^{2}-|s|^{3} \mu \phi_{y_{2}}\left(y^{*}\right) p\left(y^{*}, \nabla \phi\left(y^{*}\right)\right)\left|w_{1, \nu}\right|^{2} d \Sigma \\
& -\int_{\Sigma} \frac{1}{2}|s| \mu^{2} \phi_{y_{2}}\left(y^{*}\right)\left|\frac{\partial w_{1, \nu}}{\partial y_{2}}\right|^{2}+4|s| \mu^{2} \frac{\phi_{y_{1}}^{2}\left(y^{*}\right)}{\left|\phi_{y_{2}}\left(y^{*}\right)\right|}\left|\frac{\partial w_{1, \nu}}{\partial y_{1}}\right|^{2}+4|s| \frac{\phi_{y_{0}}^{2}\left(y^{*}\right)}{\left|\phi_{y_{2}}\left(y^{*}\right)\right|}\left|\frac{\partial w_{1, \nu}}{\partial y_{0}}\right|^{2} d \Sigma \\
& \geq C_{3} \int_{\Sigma} \frac{1}{2}|s| \mu^{2} \phi_{y_{2}}\left(y^{*}\right)\left|\frac{\partial w_{1, \nu}}{\partial y_{2}}\right|^{2}+|s|\left|\frac{\partial w_{1, \nu}}{\partial y_{1}}\right|^{2}+|s|\left|\frac{\partial w_{1, \nu}}{\partial y_{0}}\right|^{2}+\frac{1}{2}|s|^{3} \mu \phi_{y_{2}}\left(y^{*}\right) \hat{C}\left|w_{1, \nu}\right|^{2} d \Sigma .
\end{aligned}
$$

We remind that by (19) we have the equality

$$
\frac{\partial w_{2, \nu}}{\partial y_{2}}-|s| \phi_{y_{2}}\left(y^{*}\right) w_{2, \nu}=-\frac{\mu}{\lambda+2 \mu}\left(\frac{\partial w_{1, \nu}}{\partial y_{1}}-|s| \phi_{y_{1}}\left(y^{*}\right) w_{1, \nu}\right)+g_{2, \nu} .
$$


Taking the $L^{2}$ norm of the left and right hand sides of this equality and using the estimate (35) we obtain

$$
\begin{aligned}
\int_{\Sigma} h(s)\left|\frac{\partial w_{2, \nu}}{\partial y_{2}}\right|^{2}+h^{3}(s) \phi_{y_{2}}^{2}\left(y^{*}\right)\left|w_{2, \nu}\right|^{2} d \Sigma & \\
\leq C_{4}\left(\Xi_{\mu}^{(1)}+\|h(s) g\|_{\left(L^{2}(\Sigma)\right)^{2}}^{2}\right. & +\epsilon\left(\sigma_{0}\right)\left\|\left(\frac{\partial w_{1, \nu}}{\partial y_{2}}, w_{1, \nu}\right)\right\|_{X}^{2} \\
& \left.+\int_{\Sigma}\left|\frac{\partial w_{2, \nu}}{\partial y_{2}}\right|^{2}+s^{2} \phi_{y_{2}}^{2}\left(y^{*}\right)\left|w_{2, \nu}\right|^{2} d \Sigma\right),
\end{aligned}
$$

where $\epsilon\left(\sigma_{0}\right) \rightarrow 0$ as $\sigma_{0} \rightarrow 0$. By $(40),(19)$

$$
\begin{aligned}
\int_{\Sigma} h(s)\left(\left|\frac{\partial w_{2, \nu}}{\partial y_{1}}\right|^{2}+\left|\frac{\partial w_{2, \nu}}{\partial y_{0}}\right|^{2}\right) d \Sigma \leq C_{5}\left(\Xi_{\mu}^{(1)}+\|h(s) g\|_{\left(L^{2}(\Sigma)\right)^{2}}^{2}\right. & \\
& \left.+\int_{\Sigma}\left|\frac{\partial w_{2, \nu}}{\partial y_{2}}\right|^{2}+s^{2} \phi_{y_{2}}^{2}\left(y^{*}\right) w_{2, \nu}^{2} d \Sigma\right) .
\end{aligned}
$$

If we consider (30) with $\beta=\lambda+2 \mu$ then (38), (41), (42) immediately imply (18).

Now we assume that $\operatorname{supp} \mathbf{v}_{\nu} \subset \mathcal{O}\left(\delta_{1}\right)$ and $r_{\mu}(\gamma)=0$. The parameter $\delta_{1}>0$ we'll fix later. By (21)-(23) there exists $C_{6}>0$ such that

$$
\begin{aligned}
\left|\xi_{0}^{2}-s^{2} \phi_{y_{0}}^{2}\left(y^{*}\right)-\mu \xi_{1}^{2}+\mu s^{2} \phi_{y_{1}}^{2}\left(y^{*}\right)\right| & +\left|\xi_{0} s \phi_{y_{0}}\left(y^{*}\right)-\mu s \xi_{1} \phi_{y_{1}}\left(y^{*}\right)\right| \\
& \leq \delta_{1} C_{6}\left(\left|\xi_{1}\right|^{2}+\left|\xi_{0}\right|^{2}+s^{2}\right), \quad \forall \zeta \in \mathcal{O}\left(\delta_{1}\right) .
\end{aligned}
$$

Now we suppose that $\delta_{1}$ is assumed to be a sufficiently small, such that there exists a constant $C_{7}>0$ such that

$$
\left|\xi_{0}\right|^{2} \leq C_{7}\left(\left|\xi_{1}\right|^{2}+s^{2}\right) \quad \forall \zeta \in \mathcal{O}\left(\delta_{1}\right) .
$$

Next we introduce the set $\tilde{\mathcal{M}}$ by formula

$$
\begin{aligned}
\tilde{\mathcal{M}}=\left\{\zeta=\left(s, \xi_{0}, \xi_{1}\right) \in \mathcal{O}\left(\delta_{1}\right) \mid \frac{\mu}{4} \phi_{y_{2}}\left(y^{*}\right) \hat{C} s^{2}<\right. \\
\left.4 \mu^{2} \frac{\phi_{y_{1}}^{2}\left(y^{*}\right)}{\left|\phi_{y_{2}}\left(y^{*}\right)\right|} \xi_{1}^{2}+4 \frac{\phi_{y_{0}}^{2}\left(y^{*}\right)}{\left|\phi_{y_{2}}\left(y^{*}\right)\right|} \xi_{0}^{2}+\left(\left|\xi_{0}\right|^{2}+\left|\xi_{1}\right|^{2}\right)\right\} .
\end{aligned}
$$

Obviously $\mathcal{O}\left(\delta_{1}\right) \subset \mathcal{M} \cup \tilde{\mathcal{M}}$.

By (43)

$$
\left|J_{3}\right| \leq \delta_{1} \mu \phi_{y_{2}}\left(y^{*}\right)\left\|\left(\frac{\partial w_{1, \nu}}{\partial y_{2}}, w_{1, \nu}\right)\right\|_{X}^{2}
$$

Case B. Assume that

$$
\operatorname{supp} \hat{\mathbf{v}}_{\nu} \subset \tilde{\mathcal{M}}
$$

We observe that decomposition (24) with $\beta=\lambda+2 \mu$ holds true. We set $V_{\lambda+2 \mu}^{+}=$ $\left(D_{y_{2}}-\Gamma_{\lambda+2 \mu}^{+}(y, D)\right) v_{2, \nu}$. Then

$$
\mathbf{P}_{\lambda+2 \mu} v_{2, \nu}=\left(D_{y_{2}}-\Gamma_{\lambda+2 \mu}^{-}(y, D)\right) V_{\lambda+2 \mu}^{+}+T_{\lambda+2 \mu} v_{2, \nu},
$$


where $T_{\lambda+2 \mu} \in \mathcal{L}\left(H^{1}(\mathcal{Q}), L^{2}(\mathcal{Q})\right)$. This decomposition and the Proposition $1.2 \mathrm{im}$ mediately imply

$$
\begin{aligned}
&\left\|\left.h\left(D_{\sigma}\right)\left(D_{y_{2}}-\Gamma_{\lambda+2 \mu}^{+}(y, D)\right) v_{2, \nu}\right|_{y_{2}=0}\right\|_{L^{2}(\Sigma)} \\
& \quad \leq C_{5}\left(\left\|P_{\lambda+2 \mu, s} w_{2, \nu}\right\|_{L^{2}(\mathcal{Q})}+\|\mathbf{v}\|_{\left(H^{1}(\mathcal{Q})\right)^{2}}\right) .
\end{aligned}
$$

Now we need again to estimate $\Xi_{\mu}^{(1)}$. In view of (44) it suffices to estimate the term $J_{2}$.

Let us consider the equation

$$
-\frac{\mu}{\lambda+2 \mu}\left(\frac{\partial v_{1, \nu}}{\partial y_{1}}-\left|D_{\sigma}\right| \phi_{y_{1}}\left(y^{*}\right) v_{1, \nu}\right)-i \alpha_{\lambda+2 \mu}^{+}(y, D) v_{2, \nu}=i V_{\lambda+2 \mu}^{+}(\cdot, 0)-\frac{\mu}{\lambda+2 \mu} q_{2, \nu} .
$$

Since $r_{\lambda+2 \mu}(\gamma) \neq 0$ then $\alpha_{\lambda+2 \mu}^{+}(\gamma) \neq 0$. Therefore by Proposition from [T2] there exists a parametrix of the operator $\alpha_{\lambda+2 \mu}^{+}(y, D)$ which we denote as $\left(\alpha_{\lambda+2 \mu}^{+}(y, D)\right)^{-1}$. From (45) we obtain

$$
\begin{aligned}
v_{2, \nu}=-\frac{1}{i}\left(\alpha_{\lambda+2 \mu}^{+}(y, D)\right)^{-1}\left(\frac{\mu}{\lambda+2 \mu}\left(\frac{\partial v_{1, \nu}}{\partial y_{1}}-\left|D_{\sigma}\right| \phi_{y_{1}}\left(y^{*}\right) v_{1, \nu}\right)\right. \\
\left.+i V_{\lambda+2 \mu}^{+}(\cdot, 0)-\frac{\mu}{\lambda+2 \mu} q_{2, \nu}\right)+T_{0} v_{2, \nu}
\end{aligned}
$$

where $T_{0} \in \mathcal{L}\left(L^{2}(\Sigma), H^{1}(\Sigma)\right)$. Using (46), (19) we may transform $J_{2}$ to the form

$$
\begin{aligned}
& J_{2}=\operatorname{Re} \int_{\Sigma}-2\left|D_{\sigma}\right| \mu \frac{\operatorname{sign}\left(\xi_{1}^{*}\right)}{i} \sqrt{\frac{\lambda+\mu}{\lambda+2 \mu}}\left(\frac{\partial v_{1, \nu}}{\partial y_{1}}-\left|D_{\sigma}\right| \phi_{y_{1}}\left(y^{*}\right) v_{1, \nu}\right)\left(\mu \frac{\partial v_{1, \nu}}{\partial y_{1}} \phi_{y_{1}}\left(y^{*}\right)\right. \\
& \overline{\left.-\frac{\partial v_{1, \nu}}{\partial y_{0}} \phi_{y_{0}}\left(y^{*}\right)\right)} d \Sigma+R e \kappa_{3} \text {. }
\end{aligned}
$$

where

$$
\left|\kappa_{3}\right| \leq \epsilon\left\|\left(\frac{\partial w_{\nu}}{\partial y_{2}}, w_{\nu}\right)\right\|_{X}^{2}+C_{10}\left(\|h(s) g\|_{\left(L^{2}(\Sigma)\right)^{2}}^{2}+\left\|P_{\lambda+2 \mu, s} w_{2, \nu}\right\|_{L^{2}(\mathcal{Q})}^{2}\right)
$$

Since $J_{2}=R e \kappa_{3}$ we have

$$
\left|J_{2}\right| \leq \epsilon\left\|\left(\frac{\partial w_{\nu}}{\partial y_{2}}, w_{\nu}\right)\right\|_{X}^{2}+C_{11}\left(\|h(s) g\|_{\left(L^{2}(\Sigma)\right)^{2}}^{2}+\left\|P_{\lambda+2 \mu, s} w_{2, \nu}\right\|_{L^{2}(\mathcal{Q})}^{2}+\|\mathbf{v}\|_{\left(H^{1}(\mathcal{Q})\right)^{2}}^{2}\right)
$$

Next we observe that there exists $C>0$ such that

$$
\left\|\left(\frac{\partial w_{\nu}}{\partial y_{2}}, w_{\nu}\right)\right\|_{X}^{2} \leq C_{12}\left(\int_{\Sigma}\left(h(s)\left|\frac{\partial w_{1, \nu}}{\partial y_{2}}\right|^{2}+h^{3}(s)\left|w_{1, \nu}\right|^{2}\right) d \Sigma+\|h(s) g\|_{\left(L^{2}(\Sigma)\right)^{2}}^{2}\right) .
$$

Inequalities (38), (39), (44) (47), (48) imply

$$
\begin{aligned}
\left\|\left(\frac{\partial w_{\nu}}{\partial y_{2}}, w_{\nu}\right)\right\|_{X}^{2}+\left\|h(s) w_{1, \nu}\right\|_{H^{1}(\mathcal{Q})}^{2}+\left\|h^{3}(s) w_{1, \nu}\right\|_{L^{2}(\mathcal{Q})}^{2} \leq \epsilon\left\|\left(\frac{\partial w_{\nu}}{\partial y_{2}}, w_{\nu}\right)\right\|_{X}^{2}+ \\
C_{14}\left(\|\mathbf{v}\|_{\left(H^{1}(\mathcal{Q})\right)^{2}}^{2}+\left\|h(s) g_{\nu}\right\|_{\left(L^{2}(\Sigma)\right)^{2}}^{2}+\left\|P_{\mu, s} w_{2, \nu}\right\|_{L^{2}(\mathcal{Q})}^{2}\right) .
\end{aligned}
$$

From this inequality and (30) with $\beta=\lambda+2 \mu$ we obtain (18). 


\section{Case $r_{\lambda+2 \mu}=0$.}

Let $\gamma=\left(y^{*}, \zeta^{*}\right)$ be a point on $\Sigma \times S^{2}$ such that $r_{\lambda+2 \mu}(\gamma)=0$ and $\operatorname{supp} \chi_{\nu} \subset \mathcal{O}\left(\delta_{1}\right)$. We consider several cases.

Case A. Assume that

$$
s^{*}=0 \quad \text { and } \quad \lim _{\zeta \rightarrow \zeta^{*}} \operatorname{Im} r_{\mu}\left(y^{*}, \zeta\right) /|s|=0 .
$$

In that case there exists a constant $C_{2}>0$ such that

$$
-\operatorname{Im} \Gamma_{\mu}^{ \pm}(y, \zeta) \geq C_{2}|s| \quad \forall(y, \zeta) \in B_{\delta} \times \mathcal{O}\left(\delta_{1}\right)
$$

provided that $|\delta|+\left|\delta_{1}\right|$ is sufficiently small. We set $V_{\mu}^{ \pm}=\left(D_{y_{2}}-\Gamma_{\mu}^{ \pm}(y, D)\right) v_{1, \nu}$. Then

$$
\mathbf{P}_{\mu} v_{1, \nu}=\left(D_{y_{2}}-\Gamma_{\mu}^{\mp}(y, D)\right) V_{\mu}^{ \pm}+T_{\mu}^{ \pm} v_{1, \nu},
$$

where $T_{\mu}^{ \pm} \in \mathcal{L}\left(H^{1}(\mathcal{Q}), L^{2}(\mathcal{Q})\right)$. This decomposition and the Proposition 1.2 immediately imply

$$
\left\|\left.h\left(D_{\sigma}\right)\left(D_{y_{2}}-\Gamma_{\mu}^{ \pm}(y, D)\right) v_{1, \nu}\right|_{y_{2}=0}\right\|_{L^{2}(\Sigma)} \leq C_{4}\left(\left\|\mathbf{P}_{\mu} v_{1, \nu}\right\|_{L^{2}(\mathcal{Q})}+\|\mathbf{v}\|_{\left(H^{1}(\mathcal{Q})\right)^{2}}\right) .
$$

Obviously

$$
V_{\mu}^{+}(\cdot, 0)-V_{\mu}^{-}(\cdot, 0)=\left(\alpha_{\mu}^{+}(y, D)-\alpha_{\mu}^{-}(y, D)\right) v_{1, \nu} \quad \text { on } \Sigma \text {. }
$$

Since $\alpha_{\mu}^{+}\left(y^{*}, \zeta^{*}\right)-\alpha_{\mu}^{-}\left(y^{*}, \zeta^{*}\right)=2 \sqrt{r_{\mu}\left(y^{*}, \zeta^{*}\right)} \neq 0$ by (49) and Garding's inequality

$$
\int_{\Sigma}\left(h(s)\left(\left|\frac{\partial w_{1, \nu}}{\partial y_{1}}\right|^{2}+\left|\frac{\partial w_{1, \nu}}{\partial y_{0}}\right|^{2}\right)+h^{3}(s)\left|w_{1, \nu}\right|^{2}\right) d \Sigma \leq C_{5}\left(\left\|P_{\mu, s} w_{1, \nu}\right\|_{L^{2}(\mathcal{Q})}^{2}+\|\mathbf{v}\|_{\left(H^{1}(Q)\right)^{2}}^{2}\right) .
$$

From this inequality and (49) we obtain the estimate for $\frac{\partial w_{1, \nu}}{\partial y_{2}}$ :

$$
\int_{\Sigma} h(s)\left|\frac{\partial w_{1, \nu}}{\partial y_{2}}\right|^{2} d \Sigma \leq C_{6}\left(\left\|P_{\mu, s} w_{1, \nu}\right\|_{L^{2}(\mathcal{Q})}^{2}+\|\mathbf{v}\|_{\left(H^{1}(Q)\right)^{2}}^{2}\right)
$$

And finally (50), (51) combined with (19) give the estimate

$$
\left\|\left(\frac{\partial w_{2, \nu}}{\partial y_{2}}, w_{2, \nu}\right)\right\|_{X}^{2} \leq C_{7}\left(\left\|P_{\mu, s} w_{1, \nu}\right\|_{L^{2}(\mathcal{Q})}^{2}+\|\mathbf{v}\|_{\left(H^{1}(Q)\right)^{2}}^{2}+\|h(s) g\|_{\left(L^{2}(\Sigma)\right)^{2}}^{2}\right)
$$

By (50)-(52), (30), (31) we obtain (18). 
Case B. Assume that

$$
s^{*}=0 \quad \text { and } \quad \lim _{\zeta \rightarrow \zeta^{*}} \operatorname{Im} r_{\mu}\left(y^{*}, \zeta\right) /|s| \neq 0
$$

In that case first we note that since $s^{*}=0$ then $\operatorname{Re} r_{\mu}\left(y^{*}, \zeta^{*}\right)>0$. Set $\mathbf{I}=$ sign $\lim _{\zeta \rightarrow \zeta^{*}} \operatorname{Im} r_{\mu}\left(y^{*}, \zeta\right) /|s|$. For all $(y, \zeta) \in B_{\delta} \times \mathcal{O}\left(\delta_{1}\right)$ we have

$$
\Gamma_{\mu}^{+}(y, \zeta)=\mathbf{I} \sqrt{\operatorname{Re} r_{\mu}\left(y^{*}, \zeta\right)}+\tilde{b}_{1}(y, \zeta)
$$

where for the P.D.O. $\tilde{b}_{1}(y, D)$ we have the estimate

$$
\left\|h(s) \tilde{b}_{1}(y, D) w_{1, \nu}\right\|_{L^{2}(\Sigma)} \leq \epsilon\left(\delta, \delta_{1}\right)\left\|\left(\frac{\partial w_{1, \nu}}{\partial y_{2}}, w_{1, \nu}\right)\right\|_{X}^{2},
$$

where $\epsilon\left(\delta, \delta_{1}\right) \rightarrow 0$ as $|\delta|+\left|\delta_{1}\right| \rightarrow 0$.

We may assume that $|\delta|+\left|\delta_{1}\right|$ is so small that there exists $C_{8}>0$

$$
\mathbf{I} \operatorname{Im} r_{\mu}\left(y^{*}, \zeta\right) /|s| \geq C_{8}|\zeta| \quad \forall \zeta \in \mathcal{O}\left(\delta_{1}\right)
$$

Let us consider formula (32) from the previous section. One may easily see that the term $J_{3}$ is nonnegative. On the other hand (53)-(54) imply

$$
\begin{aligned}
J_{2} \geq & \int_{\Sigma} 2 \mu \sqrt{\operatorname{Re} r_{\mu}\left(y^{*}, \zeta\right)}\left|\operatorname{Im} r_{\mu}\left(y^{*}, \zeta\right)\right|\left|\hat{v}_{1, \nu}\right|^{2} d \Sigma- \\
& \quad C_{9} \epsilon\left(\delta, \delta_{1}\right)\left\|\left(\frac{\partial w_{1, \nu}}{\partial y_{2}}, w_{1, \nu}\right)\right\|_{X}^{2}-C_{10}\left(\delta, \delta_{1}\right)\left(\left\|P_{\mu, s} w_{1, \nu}\right\|_{L^{2}(\mathcal{Q})}^{2}+\|\mathbf{v}\|_{\left(H^{1}(\mathcal{Q})\right)^{2}}^{2}\right) .
\end{aligned}
$$

Inequalities (55), (56) imply

$$
\begin{aligned}
J_{2} \geq C_{11} & \int_{\Sigma} h(s)\left(\left|\frac{\partial w_{1, \nu}}{\partial y_{1}}\right|^{2}+\left|\frac{\partial w_{1, \nu}}{\partial y_{0}}\right|^{2}\right)+h^{3}(s)\left|w_{1, \nu}\right|^{2} d \Sigma \\
& -C_{9} \epsilon\left(\delta, \delta_{1}\right)\left\|\left(\frac{\partial w_{1, \nu}}{\partial y_{2}}, w_{1, \nu}\right)\right\|_{X}^{2}-C_{10}\left(\delta, \delta_{1}\right)\left(\left\|P_{\mu, s} w_{1, \nu}\right\|_{L^{2}(\mathcal{Q})}^{2}+\|\mathbf{v}\|_{\left(H^{1}(\mathcal{Q})\right)^{2}}^{2}\right) .
\end{aligned}
$$

By (56) we have that there exists a constant $C_{14}>0$

$$
\Xi_{\mu}^{(1)} \geq C_{14}\left\|\left(\frac{\partial w_{1, \nu}}{\partial y_{2}}, w_{1, \nu}\right)\right\|_{X}^{2}-C_{10}\left(\delta, \delta_{1}\right)\left(\left\|P_{\mu, s} w_{1, \nu}\right\|_{L^{2}(\mathcal{Q})}^{2}+\|\mathbf{v}\|_{\left(H^{1}(\mathcal{Q})\right)^{2}}^{2}\right) .
$$

This inequality and (19)

$$
\Xi_{\mu}^{(1)} \geq C_{15}\left\|\left(\frac{\partial w_{\nu}}{\partial y_{2}}, w_{\nu}\right)\right\|_{X}^{2}-C_{16}\left(\delta, \delta_{1}\right)\left(\left\|P_{\mu, s} w_{1, \nu}\right\|_{L^{2}(\mathcal{Q})}^{2}+\|h(s) g\|_{\left(L^{2}(\Sigma)\right)^{2}}^{2}+\|\mathbf{v}\|_{\left(H^{1}(\mathcal{Q})\right)^{2}}^{2}\right) .
$$

From (57), (30), (31) we obtain (18). 
Case C. Assume that $s^{*} \neq 0$. Then if $\delta_{1}>0$ is small enough there exists a constant $C_{17}>0$ such that

$$
\left|\xi_{0} \phi_{y_{1}}\left(y^{*}\right)-(\lambda+2 \mu) \xi_{1} \phi_{y_{1}}\left(y^{*}\right)\right|^{2} \leq \delta_{1}^{2} C_{18}\left(\left|\xi_{1}\right|^{2}+s^{2}\right) .
$$

By (30) there exists $C_{18}>0$ such that

$$
\begin{aligned}
& \Xi_{\lambda+2 \mu}^{(1)}+C_{18}\left(\left\|h(s) w_{2, \nu}\right\|_{H^{1}(\mathcal{Q})}^{2}+\left\|h^{3}(s) w_{2, \nu}\right\|_{L^{2}(\mathcal{Q})}^{2}\right) \\
& \leq C_{19}\left(\left\|\mathbf{P}_{\lambda+2 \mu} v_{2}\right\|_{L^{2}(\mathcal{Q})}^{2}+\|\mathbf{v}\|_{\left(H^{1}(\mathcal{Q})\right)^{2}}^{2}\right)+\epsilon\left\|\left(\frac{\partial w_{2, \nu}}{\partial y_{2}}, w_{2, \nu}\right)\right\|_{X}^{2}
\end{aligned}
$$

Note that $\Xi_{\lambda+2 \mu}^{(1)}$ could be written in the form

$$
\begin{aligned}
& \Xi_{\lambda+2 \mu}^{(1)}=\tilde{J}_{1}+\tilde{J}_{2}+\tilde{J}_{3}, \\
& \tilde{J}_{1}=\int_{\Sigma}|s|(\lambda+2 \mu)^{2} \phi_{y_{2}}\left(y^{*}\right)\left|\frac{\partial w_{2, \nu}}{\partial y_{2}}\right|^{2}+|s|^{3}(\lambda+2 \mu)^{2} \phi_{y_{2}}^{3}\left(y^{*}\right)\left|w_{2, \nu}\right|^{2} d \Sigma, \\
& \tilde{J}_{2}=\operatorname{Re} \int_{\Sigma} 2|s|(\lambda+2 \mu) \frac{\partial w_{2, \nu}}{\partial y_{2}} \overline{\left((\lambda+2 \mu) \phi_{y_{1}}\left(y^{*}\right) \frac{\partial w_{2, \nu}}{\partial y_{1}}-\phi_{y_{0}}\left(y^{*}\right) \frac{\partial w_{2, \nu}}{\partial y_{0}}\right)} d \Sigma \\
& \tilde{J}_{3}=\int_{\Sigma}|s|(\lambda+2 \mu) \phi_{y_{2}}\left(y^{*}\right)\left(\xi_{0}^{2}-(\lambda+2 \mu) \xi_{1}^{2}-s^{2} \phi_{y_{0}}^{2}\left(y^{*}\right)\right. \\
& \left.+s^{2}(\lambda+2 \mu) \phi_{y_{1}}^{2}\left(y^{*}\right)\right)\left|\hat{v}_{2, \nu}\right|^{2} d \Sigma \text {. }
\end{aligned}
$$

By (58), (??) we have

$$
\left|\tilde{J}_{2}+\tilde{J}_{3}\right| \leq C_{21} \delta_{1}\left\|\left(\frac{\partial w_{2, \nu}}{\partial y_{2}}, w_{2, \nu}\right)\right\|_{X}^{2}
$$

By (60) we obtain from (59) that there exists a constant $C_{22}>0$ such that

$$
\begin{aligned}
& \Xi_{\lambda+2 \mu}^{(1)} \geq-\epsilon\left\|\left(\frac{\partial w_{2, \nu}}{\partial y_{2}}, w_{2, \nu}\right)\right\|_{X}^{2} \\
& \quad+C_{22} \int_{\Sigma} h(s)(\lambda+2 \mu)^{2} \phi_{y_{2}}\left(y^{*}\right)\left|\frac{\partial w_{2, \nu}}{\partial y_{2}}\right|^{2}+h^{3}(s)(\lambda+2 \mu)^{2} \phi_{y_{2}}^{3}\left(y^{*}\right)\left|w_{2, \nu}\right|^{2} d \Sigma .
\end{aligned}
$$

From (61), inequality (49) for $V_{\mu}^{+}(\cdot, 0)$ and (19) we obtain the estimate

$$
\begin{aligned}
\Xi_{\lambda+2 \mu}^{(1)} \geq C_{27} \| & \left(\frac{\partial w_{\nu}}{\partial y_{2}}, w_{\nu}\right) \|_{X}^{2} \\
& -C_{28}\left(\delta, \delta_{1}\right)\left(\left\|P_{\mu, s} w_{1, \nu}\right\|_{L^{2}(\mathcal{Q})}^{2}+\|h(s) g\|_{\left(L^{2}(\Sigma)\right)^{2}}^{2}+\|\mathbf{v}\|_{\left(H^{1}(\mathcal{Q})\right)^{2}}^{2}\right),
\end{aligned}
$$

where $C_{27}>0$. From (62), (30), (31) we obtain (18).

\section{References}

[AK1] F. Alabau, V. Komornik. Boundary observability and controllability of linear elastodynamic systems, Optimization methods in partial differential equations, AMS, Providence (1997). 
[AK2] F. Alabau, V. Komornik. Boundary observability, controllability and stabilization of linear elastodynamic systems, SIAM J. Control Optimization 37, 521-542.

[AITY] D. Ang, M. Ikehata, D. Trong and M. Yamamoto. Unique continuation for a stationary isotropic Lamé system, Com. P.D.E 21, 371-385 (1998).

[B1] M. Bellassoued. Distribution of resonances and decay of the local energy for the elastic wave equations, Comm. Math. Phys. 215, 375-408 (2000).

[B2] M. Bellassoued. Carleman estimates and Decay Rate of the local energy for the Neumann problem of elasticity, Progr. Nonlinear Differential Equations Appl. 46,15-36 (2001).

[B3] M. Bellassoued. Unicité et contrôlle pour le système de Lamé, ESIAM 6, 561-592 (2001).

[DR] B. Dehman and L. Robbiano. La propété du prolongement unique pour un systeme elliptique le système de Lamé, J. Math. Pure Appl.72, 475-492 (1993).

[E1] Y. Egorov. Linear differential equations of the principal type, Consultants Bureau, New York (1986).

[E2] Y. Egorov. The uniqueness of the solutions of the Cauchy Problem, Dokl. Akad. Nauk. SSSR 264(4), 812-814 (1982).

[EINT] M. Eller, V. Isakov, G. Nakamura and D.Tataru. Uniqueness and stability in the Cauchy problem for Maxwell's and the elasticity system, Nonlinear Partial Differential Equations, Vol. 16, Collège de France Seminar, ElsevierGauthier Villars "Series in Applied Mathematics", Ed. P.G. Ciarlet, P.L. Lions 7, (2002).

[Hö] L. Hörmander Linear Partial Differential Operators, Springer-Verlag, Berlin (1963).

[H] M.A. Horn. Implications of sharp regularity results on boundary stabilization of the system of linear elastisity, J. Math. Analysis and Applications 223, 126-150 (1998).

[Im] O. Imanuvilov. On Carleman estimates for hyperbolic equations, to appear in Asymptotic Analysis.

[IIY] O. Imanuvilov, V. Isakov, M. Yamamoto. An inverse problem for the $d y$ namical Lamé system with two sets of boundary data, Preprint (2002).

[La] J. Lagnese. Boundary stabilization of Thin Plates, SIAM studies in Applied Mathematics (1989).

[LL] J. Lagnese and J.L. Lions. Modeling, Analysis and Control of the thin plates, Masson, Paris (1988). 
[Li] J.L. Lions. Contrôlabilite' exacte, perturbation et stabilization de systemes distribués, Vol 1, Masson, Paris, 1988.

[NW] G. Nakamura, J.-N. Wang. Unique continuation and the Runge approximation property for anisotropic elasticity, Preprint.

[T1] M. Taylor. Pseudodifferential operators, Princeton University Press, Princeton, New Jersey (1981).

[T2] M. Taylor. Pseudodifferential Operators and Nonlinear PDE, Birkhäuser, Boston Basel Berlin (1991).

[Y1] K. Yamamoto. Singularities of solutions to the boundary value problems for elastic and Maxwell's equation, Japan J. Math. 14(1),119-163 (1988).

[Y2] K. Yamamoto. Exponential energy decay of solutions of elastic wave equations with the Dirichlet condition, Math. Scand. 65, 2006-220 (1989).

[Zui] C. Zuily. Uniqueness and non-uniqueness in Cauchy problem, Birkhäuser, Boston Basel Berlin (1983).

[W1] N. Weck. Aussenraumaufgaben in der Theorie stationärer Schwingungen inhomogener elastischer Körper, Math. Z. 111, 387-398 (1969).

[W2] N. Weck. Unique continuation for systems with Lamé principal part, Math. Meth. Appl. Sci. 24, 595-605 (2001).

Department of Mathematics, Iowa State University,

400 Carver Hall, Ames IA 50011-2064 USA

vika@iastate.edu

Department of Mathematics, University of Tokyo, Komaba, Meguro, TOKYo, JaPAN

myama@ms.u-tokyo.ac.jp 